\title{
Integers without large prime factors in short intervals and arithmetic progressions
}

by

\author{
Glyn Harman (Cardiff)
}

1. Introduction. Let $\Psi(x, u)$ denote the number of integers up to $x$ having all their prime factors no more than $u$ in size. Write $\Psi(x, u ; a, q)$ for the number of such integers congruent to $a(\bmod q)$. Many authors have studied these functions ([4], [7], [9], [12], [13], [15] for example), either for their own sake, or because of possible applications (see the Royal Society Theme issue in which [9], [13] and [17] appeared). In this paper we shall be concerned with non-trivial lower bounds for $\Psi(x+y, u)-\Psi(x, u)$ and $\Psi(x, u ; a, q)$ in ranges of the parameters inaccessible to earlier workers' methods. The number $e^{-1 / 2}$ arises in all of our results (compare [2] and [8] for example), so we write $\varrho=e^{-1 / 2}$ for convenience. Note that $\varrho=0.606 \ldots$

TheOREM 1. Let $\varepsilon>0$. Then, for all large $x$, we have

$$
\Psi\left(x+x^{1 / 2}, u\right)-\Psi(x, u) \gg x^{1 / 2}
$$

provided that $u \geq x^{\varrho / 4+\varepsilon}$. The implied constant in (1.1) depends only on $\varepsilon$.

REMARKs. This shows that, for all large $x$ the interval $\left[x, x+x^{1 / 2}\right)$ contains integers with all their prime factors less than $x^{1 / 6}$ (compare the comment on page 339 of [9]). We note that our lower bound in (1.1) has the correct order of magnitude. If one increases the interval length above $x^{1 / 2}$ much stronger results have been proved (see [3], [9] and [15]), with an asymptotic formula for $\Psi(x+z, u)-\Psi(x, u)$ provided that $u \geq \exp \left((\log x)^{5 / 6+\varepsilon}\right), z \geq$ $x^{1 / 2} u^{2} \exp \left((\log x)^{1 / 6}\right)$. Of course, it is a well-known phenomenon that the quality of results for multiplicative problems in short intervals changes at this point because the more powerful Dirichlet polynomial techniques do not work for shorter interval lengths. The proof of Theorem 1 combines ideas used in discussing the greatest prime factor of the integers in $\left[x, x+x^{1 / 2}\right.$ ) (see [1] and [18] for example) with Friedlander's method in [7]. We next consider what happens for shorter intervals.

1991 Mathematics Subject Classification: Primary 11N25. 
Theorem 2. Let $y=x^{\gamma}, 3 / 7<\gamma<1 / 2$ and $\varepsilon>0$. Write

$$
\theta=\theta(\gamma)=\inf _{\kappa, \lambda} \frac{1+4 \lambda-2 \kappa-(3+4 \lambda-2 \kappa) \gamma}{4 \lambda-4 \kappa}
$$

where the infimum is taken over all known exponent pairs $(\kappa, \lambda) \neq(1 / 2,1 / 2)$. Then, if $\theta(\gamma)<\gamma$, we have

$$
\Psi(x+y, u)-\Psi(x, u) \gg y
$$

provided that $u \geq x^{\varrho \theta+\varepsilon}$. In particular, if the exponent pair conjecture, that $(\varepsilon, 1 / 2+\varepsilon)$ is an exponent pair for every $\varepsilon>0$, is true, then we can take

$$
\theta=(3-5 \gamma) / 2 \quad \text { for } 3 / 7<\gamma \leq 1 / 2 .
$$

REMARKs. Note that $\theta \rightarrow 1 / 4$ as $\gamma \rightarrow 1 / 2$ with, or without, the exponent pair conjecture. For $\gamma<1 / 2$, though, the value given by (1.4) improves on that given by (1.2) with currently known exponent pairs. Using these we have $\theta(0.45)>0.45$, but $\theta(0.46)<0.46$, so (1.3) remains valid for decreasing $y$ down to some value between $x^{0.45}$ and $x^{0.46}$. See [10] for results valid for smaller $y$.

We now move to the consideration of arithmetic progressions.

Theorem 3. Let $x>1, x^{\varepsilon}<q<x^{1 / 2-\varepsilon}, \varepsilon>0,(a, q)=1$. We have

$$
\Psi(x, u ; a, q) \gg x / q
$$

provided that

$$
\begin{array}{ll}
u>q^{\varrho / 2+\varepsilon} & \text { if } q \geq x^{3 / 7-\varepsilon}, \\
u>q^{\varrho / 3+\varepsilon} & \text { if } q \leq x^{3 / 7-\varepsilon} .
\end{array}
$$

Moreover, if $q$ is cube-free, then we can replace (1.6) by $u>q^{\varrho / 4+\varepsilon}$ for $q \leq x^{4 / 9-\varepsilon}$.

Remarks. Granville [12] proved (1.5) for

$$
u>q^{3 / 4}, \quad x \geq \max \left(u^{3 / 2+\varepsilon}, q^{3 / 4+\varepsilon}\right) .
$$

He also gave an asymptotic formula [13] for

$$
u \geq q^{1+\varepsilon} \quad \text { when } \quad \log x / \log q \rightarrow \infty .
$$

2. Outline of method. The trick in all our theorems is to count a subset of the integers under discussion, the subset being chosen to fit with our current state of arithmetical knowledge. The basic idea of Balog [3] is to count products of two numbers $m n$, and note, for any set $\mathcal{A}$,

$$
\sum_{\substack{m n \in \mathcal{A} \\ p \mid m n \Rightarrow p<x^{\alpha}}} 1 \geq \sum_{\substack{m n \in \mathcal{A} \\ p \mid m \Rightarrow p<x^{\alpha}}} 1-\sum_{\substack{m n \in \mathcal{A} \\ \exists p \mid n, p>x^{\alpha}}} 1 .
$$


Friedlander [8] gave a lower bound of the correct order of magnitude by restricting $m$ so that $p \mid m \Rightarrow p>x^{\eta}$ for some small $\eta>0$. For our theorems we need to use products of four integers, but the same basic idea is used. We distil Friedlander's method in the following result. We write $a \sim A$ to mean $A \leq a<2 A$.

Lemma 1. Let $x>1, \mathcal{B}=[x, 2 x] \cap \mathbb{Z}, \mathcal{A} \subseteq \mathcal{B}$. Let $b_{r}$ be a given sequence of non-negative reals, $b_{r} \ll 1$. Let $\alpha \in(0,1 / 2), \varepsilon>0$. Suppose that, for some $\lambda>0$ and $\eta=\varepsilon^{3}$,

$$
\sum_{\substack{r n m \in \mathcal{A} \\ m \sim M, n \sim N}} a_{n} b_{r}=\lambda \sum_{\substack{r n m \in \mathcal{B} \\ m \sim M, n \sim N}} a_{n} b_{r}+O\left(\lambda x^{1-3 \eta}\right)
$$

for any sequence $a_{n}$ such that

$$
a_{n} \ll \tau(n)
$$

and for all $M, N$ with $x^{\alpha}<M, N<x^{\alpha+\varepsilon}$. Then, writing $\beta=\alpha \varrho+\varepsilon$, we have

$$
\sum_{\substack{r k \in \mathcal{A} \\ p \mid k \Rightarrow p<x^{\beta}}} b_{r} \gg \lambda \sum_{r k \in \mathcal{B}} b_{r}+O\left(\lambda x^{1-\eta}\right) .
$$

Proof. Write $\theta=x^{-\eta}, \delta=1 / \log x$. Let $a_{n}$ be the characteristic function of the set of integers $n$ such that

$$
p \mid n \Rightarrow x^{\eta^{2}} \leq p \leq x^{\beta} .
$$

In the following we tacitly assume that the variable $r$ is restricted by

$$
\frac{1}{4} x^{1-2 \alpha-2 \varepsilon} \leq r \leq x^{1-2 \alpha} .
$$

We record here, for future reference, the result from [7] that, since $\alpha<2 \beta$,

$$
\sum_{n \leq N} a_{n}=\frac{N e^{-\gamma}}{\eta^{2} \log x}\left(1-\log \left(\frac{\alpha+\varepsilon}{\beta}\right)+O\left(\delta+\varepsilon^{3 / 2}\right)\right) .
$$

Here, and elsewhere in this lemma, $\gamma$ is Euler's constant. Clearly $a_{n}$ satisfies (2.2). We thus have, for $x^{\alpha+\eta}<M, N<x^{\alpha+\varepsilon-\eta}$,

$$
\sum_{\substack{r m n \in \mathcal{A} \\ m \sim M, n \sim N}} b_{r} a_{n}=\lambda \sum_{\substack{r m m \in \mathcal{B} \\ m \sim M, n \sim N}} b_{r} a_{n}+O(\theta x \lambda)=E(M, N) \text { say. }
$$

Hence, a lower bound for the left side of (2.3) is

$$
\frac{1}{T} \sum_{M, N}(E(M, N)-F(M, N))
$$


where

$$
F(M, N)=\sum_{\substack{r m n \in \mathcal{A} \\ m \sim M, n \sim N}} a_{n} b_{r} c_{m}, \quad c_{m}=\sum_{\substack{p \mid m \\ p \geq x^{\beta}}} 1,
$$

(note that $c_{m}$ only takes the values 0,1 since $\alpha<2 \beta$, for future convenience we insist that $c_{m}=0$ unless $x^{\alpha+\eta} \leq m \leq x^{\alpha+\varepsilon-\eta}$ )

$$
T=\max _{k \leq x} \sum_{n \mid k} a_{n} \leq 2^{\eta^{-2}},
$$

and $M, N$ are summed over values $2^{j}, 2^{k}$ with

$$
x^{\alpha+\eta}<2^{j}, 2^{k}<\frac{1}{2} x^{\alpha+\varepsilon-\eta} .
$$

Now let $d_{n}$ denote the characteristic function of all integers with no prime factor less than $x^{\eta^{2}}$. Then $d_{n} \geq a_{n}$ and, by the Fundamental Lemma of Sieve Theory (see [16] for example), there are coefficients $\lambda_{d}$ with $\left|\lambda_{d}\right| \leq 1$ such that

$$
d_{n} \leq \sum_{d \mid n} \lambda_{d}, \quad \lambda_{d}=0 \quad \text { for } d \geq x^{\eta}
$$

and

$$
\sum_{d<x^{\eta}} \frac{\lambda_{d}}{d}=\frac{e^{-\gamma}}{\eta^{2} \log x}(1+\xi)
$$

where we have written

$$
1+\xi=1+O\left(\exp \left(\frac{\log \eta}{\eta}\right)\right) .
$$

Thus

$$
\begin{aligned}
\sum_{M, N} F(M, N) & \leq \sum_{M, N} \sum_{\substack{r m n \in \mathcal{A} \\
m \sim M, n \sim N}} b_{r} c_{m} \sum_{d \mid n} \lambda_{d} \\
& =\sum_{d<x^{\eta}} \lambda_{d} \sum_{M, N} \sum_{\substack{r m l d \in \mathcal{A} \\
m \sim M, l \sim N / d}} b_{r} c_{m} \\
& =O\left(\lambda x^{1-\eta}\right)+\lambda \sum_{d<x^{\eta}} \lambda_{d} \sum_{M, N} \sum_{\substack{r m l d \in \mathcal{B} \\
m \sim M, l \sim N / d}} b_{r} c_{m}
\end{aligned}
$$

where we have applied (2.1) for each individual $d$ with $a_{d m}=c_{m}$. Now, since $\mathcal{B}=[x, 2 x]$, we have 


$$
\begin{aligned}
\sum_{\substack{r m l d \in \mathcal{B} \\
m \sim M, l \sim N / d}} b_{r} c_{m} & =\frac{1}{d} \sum_{\substack{r m n \in \mathcal{B} \\
m \sim M, n \sim N}} b_{r} c_{m}+O\left(\sum_{N m r \leq 2 x} b_{r} c_{m}\right) \\
& =\frac{1}{d} \sum_{\substack{r m n \in \mathcal{B} \\
m \sim M, n \sim N}} b_{r} c_{m}+O\left(x^{1-2 \eta}\right) .
\end{aligned}
$$

Thus

$$
\begin{aligned}
\sum_{M, N} F(M, N) & \leq \lambda \sum_{m n r \in \mathcal{B}} b_{r} c_{m} \sum_{d<x^{\eta}} \frac{\lambda_{d}}{d}+O\left(\lambda x^{1-\eta}\right) \\
& =x \sum_{r} \frac{b_{r}}{r} \sum_{m} \frac{c_{m}}{m} \cdot \frac{e^{-\gamma}}{\eta^{2} \log x}(1+\xi)+O\left(\lambda x^{1-\eta}\right)
\end{aligned}
$$

by (2.6). The main term in the upper bound is thus $\lambda$ times

$$
\begin{aligned}
\frac{e^{-\gamma}}{\eta^{2} \log x}(1+\xi) & x \sum_{r} \frac{b_{r}}{r} \sum_{x^{\beta} \leq p \leq x^{\alpha+\varepsilon}} \frac{1}{p} \sum_{x^{\alpha} / p \leq m \leq x^{\alpha+\varepsilon} / p} \frac{1}{m} \\
= & \frac{e^{-\gamma}}{\eta^{2} \log x}(1+\xi) x \sum_{r} \frac{b_{r}}{r} \sum_{x^{\beta} \leq p \leq x^{\alpha+\varepsilon}} \frac{1}{p}(\varepsilon \log x+O(1)) \\
& =\frac{e^{-\gamma}}{\eta^{2}}(1+\xi) x \sum_{r} \frac{b_{r}}{r} \log \left(\frac{\alpha+\varepsilon}{\beta}\right) .
\end{aligned}
$$

The proof is completed by noting that, using (2.4) and suppressing an error $O(\lambda \theta x)$,

$$
\sum_{M, N} E(M, N) \geq \frac{e^{-\gamma}}{\eta^{2}} \lambda\left(1-\log \left(\frac{\alpha+\varepsilon}{\beta}\right)+O\left(\delta+\varepsilon^{3 / 2}\right)\right)(\varepsilon-2 \eta) x \sum_{r} \frac{b_{r}}{r} .
$$

Thus, again suppressing an error $O(\lambda \theta x)$,

$$
\begin{aligned}
\sum_{M, N}(E(M, N) & -F(M, N)) \\
& \geq \frac{e^{-\gamma}}{\eta^{2}} \varepsilon \lambda x \sum_{r} \frac{b_{r}}{r}\left(1-2 \log \left(\frac{\alpha+\varepsilon}{\beta}\right)+O\left(\delta+\varepsilon^{3 / 2}\right)\right) .
\end{aligned}
$$

Since

$$
2 \log \left(\frac{\alpha+\varepsilon}{\beta}\right)=2 \log \left(e^{1 / 2}\right)+2 \log \left(1-\frac{\varepsilon(1-\varrho)}{\alpha \varrho+\varepsilon}\right) \leq 1-\frac{\varepsilon}{2},
$$

this gives a lower bound for the left hand side of (2.3) 


$$
\geq 2^{-1 / \eta^{2}-1} \lambda(1+O(\delta+\varepsilon)) \frac{e^{-\gamma} \varepsilon}{\eta^{2}} x \sum_{r} \frac{b_{r}}{r}+O(\lambda \theta x) \gg \lambda \sum_{r k \in \mathcal{B}} b_{r}+O(\lambda \theta x)
$$

as required.

In the following section we shall prove Theorems 1 and 2 by applying the above lemma in conjunction with exponential sum estimates. In the final section we turn to character sum estimates in order to establish Theorem 3.

3. Proof of Theorems 1 and 2 . We begin with a standard tool from Fourier analysis (compare the argument on page 39 of [11]).

Lemma 2. Let $1 \leq y \leq x^{1 / 2}, \eta>0, y x^{-\eta}<M<x$. Suppose $\left|a_{m}\right| \leq$ $\tau(m)$. Write $\mathcal{I}=[x, x+y]$. Then

$$
\sum_{\substack{m n \in \mathcal{I} \\ m \sim M}} a_{m}=y \sum_{m \sim M} \frac{a_{m}}{m}+O\left(y x^{-\eta / 2}\right)+O\left(\frac{E}{M}\right),
$$

where

$$
E=\max _{x \leq Y \leq x+y}\left|\sum_{h \leq H} \sum_{m \sim M} a_{m} e\left(\frac{Y h}{m}\right)\right|
$$

and

$$
H=M x^{\eta} / y .
$$

REMARK. The formula (3.1) is elementary for $M<y x^{-\eta}$ with the final error term removed.

Our choice of the shape of numbers to count is a consequence of the following result (Theorem 3 in [18]).

Lemma 3. Let $\eta>0,1<x \leq Y<2 x, H \leq N, N^{2} M \leq x^{1-\eta}$, $\left|a_{m}\right|,\left|b_{m}\right| \leq 1$. Also suppose that $(\kappa, \lambda)$ is an exponent pair. Then

$$
\begin{aligned}
& \left|\sum_{h \sim H} \sum_{m \sim M} a_{m} \sum_{n \sim N} b_{n} e\left(\frac{Y h}{m n}\right)\right| \\
& \ll x^{\eta}\left(\left(H M^{2} N\right)^{1 / 2}+\left(M^{3} N^{4} x^{-1}\right)^{1 / 2}\right. \\
& +\left(x^{1+2 \kappa} H^{4 \lambda} M^{4 \lambda-1-2 \kappa} N^{2-4 \kappa+4 \lambda}\right)^{1 /(2+4 \lambda)} \\
& \left.\quad+\left(x^{2 \kappa-2 \lambda} H^{2 \lambda-1} M^{6 \lambda-2 \kappa} N^{10 \lambda+1-4 \kappa}\right)^{1 /(2+4 \lambda)}\right) .
\end{aligned}
$$

REMARK. For Theorem 1 the older Lemma 14 of [1] would have sufficed in place of the above. The bound (3.3) has an advantage in our proof when $y<x^{1 / 2}$.

Proof of Theorem 1. We first require the following result on exponent pairs. We refer the reader to [11] for an introduction to this subject. 
LEMMA 4. The exponent pair $B A^{n} B, n \geq 1$, has the form

$$
(\kappa, \lambda)=(1 / 2-(n+1) \delta, 1 / 2+\delta)
$$

with

$$
\frac{1}{2} \cdot 3^{-n} \leq \delta \leq \frac{1}{3} \cdot 2^{-n} .
$$

REMARK. It will be vital that

$$
\frac{1 / 2-\kappa}{\lambda-1 / 2} \rightarrow \infty \quad \text { and } \quad \delta \rightarrow 0 \quad \text { as } n \rightarrow \infty .
$$

Proof (of Lemma 4). The result follows quickly by induction from the definition of the $A$ and $B$ processes:

$$
A(\kappa, \lambda)=\left(\frac{\kappa}{2 \kappa+2}, \frac{\kappa+\lambda+1}{2 \kappa+2}\right), \quad B(\kappa, \lambda)=\left(\lambda-\frac{1}{2}, \kappa+\frac{1}{2}\right) .
$$

We now show that it is possible to estimate

$$
\sum_{\substack{l m n \in \mathcal{I} \\ m \sim M, n \sim N}} a_{m} b_{n}
$$

when $M N \leq x^{3 / 4-\varepsilon}$ for a certain choice of $M$ with $y=x^{1 / 2}$. To do this we need only prove that the right hand side of $(3.3)$ is $O\left(M N x^{-\eta}\right)$ for $H \leq M N x^{\eta} / y$. We put $M=y x^{-5 \eta}$, and take $H$ at its maximum value which will thus be $N x^{-4 \eta}$. We thus have $H \leq N$ and $N^{2} M \leq x^{1-\eta}$ if $\varepsilon>3 \eta$. We have

$$
x^{\eta}\left(H M^{2} N\right)^{1 / 2} \leq x^{\eta}\left(M^{2} N^{2} x^{-4 \eta}\right)^{1 / 2}=M N x^{-\eta},
$$

as required. Also

$$
\begin{aligned}
\left(M^{3} N^{4} x^{-1}\right)^{1 / 2} x^{\eta} & =M N\left(M N^{2} x^{-1+2 \eta}\right)^{1 / 2}=M N\left(\frac{M^{2} N^{2}}{x} \cdot \frac{x^{2 \eta}}{M}\right) \\
& \leq M N\left(x^{1 / 2-2 \varepsilon} x^{-1 / 2+7 \eta}\right)^{1 / 2} \leq M N x^{-\eta}
\end{aligned}
$$

assuming that $2 \varepsilon \geq 9 \eta$.

The next term is the trickiest to estimate. We need to establish that

$$
x^{1+2 \kappa} N^{4 \lambda} x^{-4 \lambda \eta} M^{4 \lambda-1-2 \kappa} N^{2-4 \kappa+4 \lambda} \leq(M N)^{2+4 \lambda} x^{-2 \eta(2+4 \lambda)} .
$$

The inequality (3.5) is equivalent to

$$
N^{4 \lambda-4 \kappa} \leq M^{3+2 \kappa} x^{-1-2 \kappa+4 \lambda \eta-2 \eta(2+4 \lambda)},
$$

that is,

$$
N \leq\left(x^{3 / 2+\kappa-1-2 \kappa+4 \lambda \eta-2 \eta(2+4 \lambda)-5(3+2 \kappa) \eta}\right)^{1 /(4 \lambda-4 \kappa)},
$$

or

$$
M N \leq\left(x^{1 / 2+2 \lambda-3 \kappa-5 \eta(3-2 \kappa+4 \lambda)+4 \lambda \eta-2 \eta(2+4 \lambda)}\right)^{1 /(4 \lambda-4 \kappa)} .
$$


Now suppose that $(\kappa, \lambda)=(1 / 2-(n+1) \delta, 1 / 2+\delta)$. Then the right hand side of (3.6) can be written $X_{1} X_{2}$ where $X_{1}$ does not depend on $\eta$ and $X_{2}=x^{-\eta c}$ where $c$ does not depend on $\eta$. We have

$$
X_{1}=x^{(3 n+5) /(4 n+8)} .
$$

We may therefore choose $n$ sufficiently large so that $X_{1} \geq x^{3 / 4-\varepsilon / 2}$. We may then pick $\eta$ sufficiently small so that $X_{2} \geq x^{-\varepsilon / 2}$. Thus $M N \leq x^{3 / 4-\varepsilon}$ suffices as claimed. The reader will quickly verify that the final term in (3.3) is smaller than the term we have just estimated.

Now we count numbers $l k u v \in \mathcal{I}$ with

$$
\begin{aligned}
x^{1 / 4+\varepsilon} & \leq l \leq x^{1 / 4+2 \varepsilon}, \quad x^{1 / 4+\varepsilon} \leq v \leq x^{1 / 4+2 \varepsilon}, \\
u v \sim M & =x^{1 /(2-5 \eta)}, \quad p \mid k u \Rightarrow x^{1 / 10} \leq p \leq x^{\varrho / 4} .
\end{aligned}
$$

By our above working we can give the correct asymptotic formula with any reasonable sequence $a_{m}$ for

$$
\sum_{\substack{l k k u v \in \mathcal{I} \\ l \sim L, u v \sim M}} a_{v} c_{k}
$$

where $c_{k}$ is the characteristic function of those integers such that $p \mid n \Rightarrow$ $x^{1 / 10} \leq p \leq x^{\varrho / 4}$. The result follows from Lemma 1 with $k u=r, v=n$, $l=m, \lambda=x^{-1 / 2}$. We here use the fact that

$$
b_{r}=\sum_{k u=r} c_{k}<32,
$$

since $r<x^{1 / 2}$ while $p \mid k u \Rightarrow p>x^{1 / 10}$. Also we noted that

$$
\sum_{r k \in \mathcal{B}} b_{r} \gg x
$$

Proof of Theorem 2. In place of (3.7) we have

$$
\left(y^{3+4 \lambda-2 \kappa} x^{-1-2 \kappa}\right)^{1 /(4 \lambda-4 \kappa)} \text {. }
$$

This is $x^{1-\theta}$ with

$$
\theta=\frac{1+4 \lambda-2 \kappa-(3+4 \lambda-2 \kappa) \gamma}{4 \lambda-4 \kappa} .
$$

Now we take the infimum of this expression over all exponent pairs $(\kappa, \lambda)$. The value we eventually obtain for the variable $u$ is $x^{\varrho \theta+\varepsilon}$ by the above construction, provided that $\theta(\gamma)<\gamma$. This last condition being necessary since we require $v<y x^{-5 \eta}$ in the proof. The reader should have no trouble completing the proof as before. 
4. Proof of Theorem 3. We first require two lemmas.

LemmA 4. Let $1<q<x^{1 / 2-\varepsilon},(a, q)=1$. Write $\eta=\varepsilon^{2}$. Suppose that $M, N, R$ satisfy

$$
q^{1 / 2} x^{\eta} \leq N \leq q^{1 / 2} x^{2 \eta}, \quad q^{1 / 2} \leq R \leq q^{1 / 2} x^{\eta}, \quad M N R=x / 8 .
$$

Then, for any coefficients $a_{m}, c_{r}$ with $\left|a_{m}\right| \ll \tau(m),\left|c_{r}\right| \ll \tau(r)$, we have

$$
\sum_{\substack{m n r \equiv a \bmod q \\ m \sim M, n \sim N, r \sim R}} a_{m} c_{r}=\frac{1}{\phi(q)} \sum_{\substack{(m r n, q)=1 \\ m \sim M, n \sim N, r \sim R}} a_{m} c_{r}+O\left(x^{1-\eta / 4} q^{-1}\right) .
$$

Proof. We have the left hand side of (4.2) equal to

$$
\begin{aligned}
\frac{1}{\phi(q)} \sum_{\chi \bmod q} \bar{\chi}(a) \sum_{m \sim M} a_{m} \chi(m) & \sum_{n \sim N} \chi(n) \sum_{r \sim R} c_{r} \chi(r) \\
& =\frac{1}{\phi(q)} \sum_{\chi \bmod q} \bar{\chi}(a) A(\chi) B(\chi) C(\chi) \quad \text { say. }
\end{aligned}
$$

The term $\chi=\chi_{0}$ gives the main term on the right of (4.2). We use Hölder's inequality on the remaining terms to obtain an error

$$
\begin{aligned}
\leq & \left(\frac{1}{\phi(q)} \sum_{\chi \neq \chi_{0}}|A(\chi)|^{2}\right)^{1 / 2}\left(\frac{1}{\phi(q)} \sum_{\chi \neq \chi_{0}}|B(\chi)|^{4}\right)^{1 / 4} \\
& \times\left(\frac{1}{\phi(q)} \sum_{\chi \neq \chi_{0}}|C(\chi)|^{4}\right)^{1 / 4} .
\end{aligned}
$$

We note that

$$
\frac{1}{\phi(q)} \sum_{\chi \bmod q}\left|\sum_{d \sim D} b_{d} \chi(d)\right|^{2} \leq\left(1+\frac{D}{q}\right) \sum_{d \sim D}\left|b_{d}\right|^{2}
$$

(see [18], Theorem 6.2) and

$$
\frac{1}{\phi(q)} \sum_{\chi \neq \chi_{0}}\left|\sum_{n \sim N} \chi(n)\right|^{4} \ll q^{\eta} N^{2}
$$

(see [14], Lemma 2). We thus obtain an error

$$
\begin{aligned}
& \ll x^{\eta / 2} q^{\eta / 4}\left(\left(\frac{x}{M N R}\right)^{2} \frac{1}{q}\right)^{1 / 2}\left(\frac{R^{4}}{q}\right)^{1 / 4} N^{1 / 2} \\
& \ll x^{3 \eta / 4} \frac{x}{q}\left(\frac{q}{N^{2}}\right)^{1 / 2} \leq \frac{x^{1-\eta / 4}}{q}
\end{aligned}
$$

as required. 
Lemma 5. Given the hypotheses of Lemma 4 except that $1<q<x^{3 / 7}$ and $M, N, R$ satisfy

(4.4) $\quad q^{1 / 3} x^{\eta} \leq N \leq q^{1 / 3} x^{2 \eta}, \quad(x / N)^{1 / 2} \leq R \leq(x / N)^{1 / 2} x^{\eta}, \quad M N R=x / 8$. Then (4.2) holds. Moreover, if $q$ is cube-free with $1<q<x^{4 / 9}$ then one can replace (4.4) with

(4.5) $\quad q^{1 / 4} x^{\eta} \leq N \leq q^{1 / 4} x^{2 \eta}, \quad(x / N)^{1 / 2} \leq R \leq(x / N)^{1 / 2} x^{\eta}, \quad M N R=x / 8$.

In this last case the error in (4.2) becomes $x^{1-\eta^{2} / 4} / q$.

Proof. Instead of Hölder's inequality in the last proof we use Cauchy's inequality to bound the error as

$$
\leq\left(\frac{1}{\phi(q)} \sum_{\chi \neq \chi_{0}}|A(\chi)|^{2}\right)^{1 / 2}\left(\frac{1}{\phi(q)} \sum_{\chi \neq \chi_{0}}|C(\chi)|^{2}\right)^{1 / 2} \max _{\chi \neq \chi_{0}}|B(\chi)| .
$$

We now use the Burgess bound (see [5]), that

$$
|B(\chi)| \ll q^{1 / 9} N^{2 / 3+\delta}
$$

for any $\delta>0$, to bound (4.6) as

$$
\ll x^{\eta / 12} q^{1 / 9} N^{2 / 3}\left(\left(\frac{x}{N R}\right)^{2} \frac{1}{q}\right)^{1 / 2}\left(\frac{R^{2}}{q}\right)^{1 / 2}
$$

(noting that both of $x /(N R), R$ exceed $q$ by (4.4)),

This completes the proof for general $q$.

$$
\ll \frac{x^{1+\eta / 12}}{q} N^{-1 / 3} q^{1 / 9} \leq \frac{x^{1-\eta / 4}}{q} .
$$

For $q$ cube-free we note that the Burgess bound can be improved to

$$
|B(\chi)| \ll q^{\eta / 4+\eta^{2}} N^{1-\eta} \leq N x^{-\eta^{2} / 2}
$$

(take $k=\left[\eta^{-1}\right]$ in [5] with $\varepsilon=\eta^{2} / 2$ ). This suffices to obtain the claimed result.

Proof of Theorem 3. We consider numbers in an analogous way to Theorem 1:

with

$$
l k u v \equiv a(\bmod q)
$$

$$
\begin{gathered}
q^{1 / 2} x^{\eta} \leq l, v \leq q^{1 / 2} x^{2 \eta}, \quad q^{1 / 2} \leq k \leq q^{1 / 2} x^{\eta} \\
l k u v \leq x, \quad p \mid k u \Rightarrow q^{\varrho / 8} \leq p \leq q^{\varrho / 2} .
\end{gathered}
$$

The proof may then be easily completed for $u>q^{\varrho / 2+\varepsilon}$. For $q<x^{3 / 7-\varepsilon}$ the choice of variable size becomes

$$
q^{1 / 3} x^{\eta} \leq l, v \leq q^{1 / 3} x^{2 \eta}, \quad\left(\frac{x}{l v}\right)^{1 / 2} \leq k \leq\left(\frac{x}{l v}\right)^{1 / 2} x^{\eta},
$$

while the cube-free case requires $q^{1 / 3}$ to be replaced by $q^{1 / 4}$ in the above. 


\section{References}

[1] R. C. Baker, The greatest prime factor of the integers in an interval, Acta Arith. 47 (1986), 193-231.

[2] R. C. Baker and G. Harman, Shifted primes without large prime factors, ibid. 83 (1998), 331-361.

[3] A. Balog, $p+a$ without large prime factors, Sém. Théorie des Nombres Bordeaux (1983-84), exposé 31.

[4] A. Balog and C. Pomerance, The distribution of smooth numbers in arithmetic progressions, Proc. Amer. Math. Soc. 115 (1992), 33-43.

[5] D. A. Burgess, On character sums and L-series, II, Proc. London Math. Soc. (3) 13 (1963), 525-536.

[6] - , The character sum estimate with $r=3$, J. London Math. Soc. (2) 33 (1986), 219-226.

[7] J. B. Friedlander, Integers free from large and small primes, Proc. London Math. Soc. (3) 33 (1976), 565-576.

[8] - Shifted primes without large prime factors, in: Number Theory and Applications, 1989, Kluwer, Berlin, 1990, 393-401.

[9] J. B. Friedlander and A. Granville, Smoothing 'smooth' numbers, Philos. Trans. Roy. Soc. London Ser. A 345 (1993), 339-347.

[10] J. B. Friedlander and J. C. Lagarias, On the distribution in short intervals of integers having no large prime factor, J. Number Theory 25 (1987), 249-273.

[11] S. W. Graham and G. Kolesnik, Van der Corput's Method of Exponential Sums, London Math. Soc. Lecture Note Ser. 126, Cambridge Univ. Press, 1991.

[12] A. Granville, Integers, without large prime factors, in arithmetic progressions I, Acta Math. 170 (1993), 255-273.

[13] - Integers, without large prime factors, in arithmetic progressions II, Philos. Trans. Roy. Soc. London Ser. A 345 (1993), 349-362.

[14] G. Harman, Diophantine approximation with square-free integers, Math. Proc. Cambridge Philos. Soc. 95 (1984), 381-388.

[15] —, Short intervals containing numbers without large prime factors, ibid. 109 (1991), $1-5$.

[16] H. Iwaniec, Rosser's sieve, Acta Arith. 36 (1980), 171-202.

[17] H. W. Lenstra, Jr., J. Pila and C. Pomerance, A hyperelliptic smoothness test I, Philos. Trans. Roy. Soc. London Ser. A 345 (1993), 397-408.

[18] H.-Q. Liu and J. Wu, Numbers with a large prime factor, Acta Arith. 89 (1999), 163-187.

[19] H. L. Montgomery, Topics in Multiplicative Number Theory, Springer, 1971.

School of Mathematics

Cardiff University

P.O.Box No. 926

Cardiff CF2 4YH

Wales, U.K.
Present address:

Mathematics Department Royal Holloway

University of London

Egham, Surrey TW20 OEX

England

E-mail: G.Harman@rhbnc.ac.uk 